PROCEEDINGS OF THE

AMERICAN MATHEMATICAL SOCIETY

Volume 130, Number 9, Pages 2711-2717

S 0002-9939(02)06398-0

Article electronically published on April 17, 2002

\title{
FOURIER ASYMPTOTICS OF CANTOR TYPE MEASURES AT INFINITY
}

\author{
TIAN-YOU HU AND KA-SING LAU
}

(Communicated by Andreas Seeger)

\begin{abstract}
Let $q \geq 3$ be an integer and let $\phi(t)=\prod_{n=1}^{\infty} \cos \left(q^{-n} t\right)$. In this note we prove that $\lim _{t \rightarrow \infty} \phi(t)=-\phi(\pi)$ for all $q$; $\varlimsup_{t \rightarrow \infty} \phi(t)=\phi(\pi)$ if $q$ is odd and $\varlimsup_{t \rightarrow \infty} \phi(t) \leq \phi(\pi)$ if $q$ is even. This improves a classical result of Wiener and Wintner. We also give a necessary and sufficient condition for the product $\prod_{i=1}^{m} \phi\left(\alpha_{i} t\right)$ to approach zero at infinity.
\end{abstract}

\section{INTRODUCTION}

In the celebrated paper [WW] Wiener and Wintner proved that if

$$
\phi(t)=\prod_{n=1}^{\infty} \cos \left(q^{-n} t\right)
$$

where $q \geq 3$ is an integer, then $\varlimsup_{t \rightarrow \infty} \phi(t)>0$. Moreover, the average $\frac{1}{2 T} \int_{-T}^{T}|\phi(t)|^{2} d t=O\left(T^{\frac{\log 2}{\log q}}\right)$, as $T \rightarrow \infty$. Note that $\phi$ is the characteristic function of the random variable $X=\sum_{n=1}^{\infty} \rho^{n} \varepsilon_{n}$, where $\left\{\varepsilon_{n}\right\}_{n=1}^{\infty}$ is a sequence of i.i.d. Bernoulli random variables, and the corresponding distribution is a Cantor type measure. This measure is the most basic model in fractal theory; it is generated by the contractions $S_{1} x=\rho x, S_{2} x=\rho x+1,0<\rho<1 / 2$. The above result of Wiener and Wintner has been extended by Strichartz Str1, 2] and Lau and Wang [LW] to more general self-similar measures generated by similitudes $\left\{S_{i}\right\}_{i=1}^{m}$ that satisfy the open set condition. Fan and Lau [FL] replaced the cosine function in the infinite product by a periodic function and investigated its multifractal structure at infinity. In another direction, Liu [L, Theorem 2.1] has found that the exact values of $\varlimsup_{t \rightarrow \infty} \phi(t)$ and $\varlimsup_{t \rightarrow \infty} \phi_{\alpha}(t)$ (to be defined in the sequel) are useful to determine the solutions of the distributional equations arise from some random multiplicative cascade. Motivated by this, we investigate the limit extrema of the above expressions. We prove

Theorem 1.1. Let $q \geq 3$ be an integer and let $\phi(t)=\prod_{n=1}^{\infty} \cos \left(q^{-n} t\right)$. Then $\varliminf_{t \rightarrow \infty} \phi(t)=-\phi(\pi)$ for all $q, \varlimsup_{t \rightarrow \infty} \phi(t)=\phi(\pi)$ if $q$ is odd, and $\varlimsup_{t \rightarrow \infty} \phi(t) \leq$ $\phi(\pi)$ if $q$ is even.

Received by the editors February 4, 2001 and, in revised form, April 20, 2001.

2000 Mathematics Subject Classification. Primary 42A38; Secondary 26A12.

Key words and phrases. Cantor type measure, Fourier transform.

Research supported by an HKRGC grant. 
We do not know the exact value of $\varlimsup_{t \rightarrow \infty} \phi(t)$ when $q$ is even. In fact, at the end of the note we display a graph of $\phi(t)$ for $q=4$ which suggests that $\varlimsup_{t \rightarrow \infty} \phi(t)<\phi(\pi)$.

For $\left\{\alpha_{1}, \cdots, \alpha_{m}\right\} \subseteq \mathbb{R}^{+}$, let

$$
\phi_{\alpha}(t)=\prod_{i=1}^{m} \phi\left(\alpha_{i} t\right)=\prod_{i=1}^{m} \prod_{n=1}^{\infty} \cos \left(q^{-n} \alpha_{i} t\right) .
$$

Note that $\phi_{\alpha}$ is the Fourier transform of the measure $\mu$ induced by $X=\sum_{i=1}^{m} \alpha_{i} X_{i}$, where $\left\{X_{i}\right\}_{i=1}^{m}$ are i.i.d. random variables, each induced by the $\frac{1}{q}$-Cantor measure. We rewrite $X$ as $X=\sum_{n=1}^{\infty} q^{-n} Y_{n}$, where $\left\{Y_{n}\right\}_{n=1}^{\infty}$ are i.i.d. and $Y_{n} \sim \sum_{i=1}^{m} \alpha_{i} \varepsilon_{i}$. From this expression we see that $\mu$ is the self-similar measure generated by $S_{j} x=$ $x / q+b_{j}$, where $b_{j}=\sum_{i=1}^{m} \alpha_{i} \varepsilon_{i}, \varepsilon_{i}=0$ or 1 and the weight associated with the map is $w_{j}=2^{-m} \#\left\{\left(\varepsilon_{1}, \cdots, \varepsilon_{m}\right): \sum_{i=1}^{m} \alpha_{i} \varepsilon_{i}=b_{j}, \varepsilon_{i}=0\right.$ or 1$\}$.

A set $\left\{\alpha_{1}, \cdots, \alpha_{m}\right\}$ of positive numbers is called commensurable if for all $1 \leq i, j \leq m, \alpha_{i} \alpha_{j}^{-1}$ are rational numbers, and incommensurable otherwise. If $\left\{\alpha_{1}, \cdots, \alpha_{m}\right\}$ is commensurable, then it is easy to show that there is an $a>0$ such that

$$
\alpha_{i}=a N_{i}, \quad i=1, \cdots, m,
$$

where the $N_{i}$ 's are positive integers and have no common divisor. In the following we need to use a special expression of $N_{i}$ by writing

$$
N_{i}=q^{d_{i}} n_{i}
$$

with $d_{i} \in \mathbb{N}$. We also write

$$
n_{i}=l_{i} q+r_{i}
$$

where $l_{i}, r_{i} \in \mathbb{N}$, with $1 \leq r_{i} \leq q-1$.

Theorem 1.2. Let $q \geq 3$ be an integer and let $\phi_{\alpha}(t)$ be defined as above.

(i) If $\left\{\alpha_{1}, \cdots, \alpha_{m}\right\}$ is incommensurable, then $\overline{\lim }_{t \rightarrow \infty} \phi_{\alpha}(t)=0$.

(ii) Suppose that $\left\{\alpha_{1}, \cdots, \alpha_{m}\right\}$ is commensurable. If $q$ is even and if $r_{j}=q / 2$ for some $j$, where $r_{j}$ is as in (1.3), then $\varlimsup_{t \rightarrow \infty} \phi_{\alpha}(t)=0$. Otherwise,

$$
\varlimsup_{t \rightarrow \infty}\left|\phi_{\alpha}(t)\right|=\sup \left\{\prod_{i=1}^{m}\left|\phi\left(s n_{i} \pi\right)\right|: s=1,2, \ldots\right\}>0
$$

where the $n_{i}$ 's are as in (1.3).

\section{The PRoofs}

It is easy to see that $\phi(t)$ satisfies the equation

$$
\phi\left(q^{n} t\right)=\phi(t) \prod_{i=0}^{n-1} \cos \left(q^{i} t\right) .
$$

By replacing $t$ with $q^{-n} t$, we have

$$
\phi(t)=\phi\left(q^{-n} t\right) \prod_{i=1}^{n} \cos \left(q^{-i} t\right) .
$$


By using (2.2) and $\phi(0)=1$, it follows that

$$
\phi(t)=0 \quad \text { iff } \quad t=q^{i}\left(l+\frac{1}{2}\right) \pi \quad \text { for some } i>0, l \in \mathbb{Z} .
$$

In order to prove the two theorems in Section 1, we need a few lemmas for $\phi_{\alpha}(t)=$ $\prod_{i=1}^{m} \phi\left(\alpha_{i} t\right)$. Let

$$
Q=\left\{k q^{-j} \pi: q \nmid k, k, j \in \mathbb{N}\right\} .
$$

Lemma 2.1. Let $q \geq 3$ and let $\alpha_{i}=a q^{d_{i}} n_{i}$ be defined by (1.1)-(1.3).

(i) If there is a $t>0$ such that $\alpha_{i} t=k_{i} q^{-j_{i}} \pi \in Q$ for all $i=1, \cdots, m$, then there is a positive integer $s$ such that $k_{i}=s n_{i}$ for all $i=1, \cdots, m$. Furthermore,

(a) if $k_{i} \neq q^{k}\left(l+\frac{1}{2}\right)$ for all $k, l \in \mathbb{N}, i=1, \cdots, m$, then

$$
\left|\phi_{\alpha}\left(q^{n} t\right)\right|=\prod_{i=1}^{m}\left|\phi\left(s n_{i} \pi\right)\right|>0 \quad \text { for all } n>\max _{1 \leq i \leq m}\left\{j_{i}\right\}
$$

(b) if there is a $k_{i}=q^{k}\left(l+\frac{1}{2}\right)$ for some $k, l \in \mathbb{N}$, then $\left|\phi_{\alpha}\left(q^{n} t\right)\right|=0$ for all $n>j_{i}$.

(ii) If there are $t>0$ and $i \in \mathbb{N}$ such that $\alpha_{i} t \notin Q$, then $\varlimsup_{n \rightarrow \infty}\left|\phi_{\alpha}\left(q^{n} t\right)\right|=0$.

Proof. (i) Since $\alpha_{i} t=k_{i} q^{-j_{i}} \pi$ and $\alpha_{i}=a q^{d_{i}} n_{i}$ for all $i$, it follows that for $1 \leq i, l \leq$ $m$,

$$
\frac{k_{i}}{n_{i}}=\frac{k_{l}}{n_{l}} q^{k_{i, l}}, \quad \text { where } k_{i, l} \in \mathbb{Z}
$$

It is easy to see that $k_{i, l}=0$, since $q \nmid k_{i}$ and $q \nmid n_{i}$ for all $i=1, \cdots, m$. Hence there are two co-prime integers $n$ and $s$ such that for $1 \leq i, l \leq m$,

$$
\frac{k_{i}}{n_{i}}=\frac{k_{l}}{n_{l}}=\frac{s}{n} \text {. }
$$

Therefore $k_{i}=s n_{i} / n$, and hence $n \mid n_{i}$ for all $i=1, \cdots, m$. This forces $n$ to be 1 , so that $k_{i}=s n_{i}$ for $i=1, \cdots, m$, as asserted.

To prove (a), we see that by $(2.1),\left|\phi\left(q^{n} k \pi\right)\right|=|\phi(k \pi)|$ for $k, n \in \mathbb{N}$. So for $n>\max \left\{j_{i}: 1 \leq i \leq m\right\}$, we have

$$
\left|\phi_{\alpha}\left(q^{n} t\right)\right|=\left|\prod_{i=1}^{m} \phi\left(q^{n} \alpha_{i} t\right)\right|=\left|\prod_{i=1}^{m} \phi\left(q^{n-j_{i}} k_{i} \pi\right)\right|=\left|\prod_{i=1}^{m} \phi\left(k_{i} \pi\right)\right|=\left|\prod_{i=1}^{m} \phi\left(s n_{i} \pi\right)\right| .
$$

By (2.3), the above product is positive if and only if $k_{i} \neq q^{k}\left(l+\frac{1}{2}\right)$ for all $k, l \in \mathbb{N}$, $i=1, \cdots, m$. This proves (a), and also (b).

To show (ii), we first prove that if $t \notin Q$, then $\varlimsup_{n \rightarrow \infty}\left|\phi\left(q^{n} t\right)\right|=0$. By (2.1) it suffices to show that there exists $0<\eta<1$ such that $\left|\cos \left(q^{n} t\right)\right| \leq \eta$ for infinitely many $n \in \mathbb{N}$.

For $x \geq 0$, let $\|x\|=\min \{|x-k \pi|: k \in \mathbb{N}\}$. Let $\eta$ be such that $\cos \left(\frac{\pi}{q+1}\right)<$ $\eta<1$. For $t \notin Q$ and for any $n$, if $\left\|q^{n} t\right\| \geq \frac{\pi}{q+1}$, then $\left|\cos \left(q^{n} t\right)\right| \leq \cos \left(\frac{\pi}{q+1}\right)<\eta$. If $\left\|q^{n} t\right\|<\frac{\pi}{q+1}$, we write $q^{n} t=(k+\varepsilon) \pi$, where $0<|\varepsilon|<\frac{1}{q+1}$. There exists $j$ such that $\frac{1}{q+1} \leq q^{j}|\varepsilon| \leq \frac{q}{q+1}$. This implies that $\left\|q^{n+j} t\right\| \geq \frac{\pi}{q+1}$, and hence $\left|\cos \left(q^{n+j} t\right)\right| \leq\left|\cos \left(\frac{\pi}{q+1}\right)\right|<\eta$.

If $\alpha_{i} t \notin Q$ for some $i$, then

$$
\varlimsup_{n \rightarrow \infty}\left|\phi_{\alpha}\left(q^{n} t\right)\right|=\varlimsup_{n \rightarrow \infty}\left|\prod_{i=1}^{m} \phi\left(q^{n} \alpha_{i} t\right)\right|=0 .
$$


Lemma 2.2. $\varlimsup_{x \rightarrow \infty}\left|\phi_{\alpha}(x)\right|=\sup \left\{\varlimsup_{n \rightarrow \infty}\left|\phi_{\alpha}\left(q^{n} t\right)\right|: t>0\right\}$.

Proof. It suffices to show that the left hand side is less than or equal to the right hand side. Let $t_{n} \rightarrow \infty$ be such that $\lim _{n \rightarrow \infty}\left|\phi_{\alpha}\left(t_{n}\right)\right|=\lambda>0$. We will show that there exists $t>0$ such that $\varlimsup_{n \rightarrow \infty}\left|\phi_{\alpha}\left(q^{n} t\right)\right| \geq \lambda$.

Let $\left\{k_{n}\right\}_{n=1}^{\infty}$ be such that $q^{-k_{n}} t_{n} \in\left[\frac{\pi}{q}, \pi\right]$. Without loss of generality we assume that $\lim _{n \rightarrow \infty} q^{-k_{n}} t_{n}=t$.

We claim that $\varlimsup_{n \rightarrow \infty}\left|\phi_{\alpha}\left(q^{n} t\right)\right|=\gamma \geq \lambda$. Otherwise $\gamma+\varepsilon<\lambda$ for some $\varepsilon>0$. We can find $N$ such that for all $n \geq N,\left|\phi_{\alpha}\left(q^{n} t\right)\right|<\gamma+\frac{\varepsilon}{2}$. Note that $\phi_{\alpha}\left(q^{N} t\right)$ is a continuous function of $t$; hence, for large $n$,

$$
\left|\phi_{\alpha}\left(q^{N-k_{n}} t_{n}\right)-\phi_{\alpha}\left(q^{N} t\right)\right|<\frac{\varepsilon}{2} .
$$

Since (2.2) implies that $\left|\phi_{\alpha}(t)\right| \leq\left|\phi_{\alpha}\left(q^{-n} t\right)\right|$ for all $n$, for large $n$ we get

$$
\left|\phi_{\alpha}\left(t_{n}\right)\right| \leq\left|\phi_{\alpha}\left(q^{N-k_{n}} t_{n}\right)\right|<\frac{\varepsilon}{2}+\left|\phi_{\alpha}\left(q^{N} t\right)\right|<\gamma+\varepsilon,
$$

so that $\lim _{n \rightarrow \infty}\left|\phi_{\alpha}\left(t_{n}\right)\right| \leq \gamma+\varepsilon<\lambda$. The contradiction proves the lemma.

Corollary 2.1. $\varlimsup_{x \rightarrow \infty}\left|\phi_{\alpha}(x)\right|=0$ if and only if $\varlimsup_{n \rightarrow \infty}\left|\phi_{\alpha}\left(q^{n} t\right)\right|=0$ for every $t>0$.

Corollary 2.2. If $\varlimsup_{x \rightarrow \infty}\left|\phi_{\alpha}(x)\right|=\lambda>0$, then $\left\{\alpha_{1}, \cdots, \alpha_{m}\right\}$ is commensurable and

$$
\lambda=\sup \left\{\prod_{i=1}^{m}\left|\phi\left(s n_{i} \pi\right)\right|: s=1,2, \cdots\right\},
$$

where $n_{i}$ is related to $\alpha_{i}$ through (1.1)-(1.3).

Proof. For any $\varepsilon>0$, by Lemma 2.2 there is $t>0$ such that $\lim _{n \rightarrow \infty}\left|\phi_{\alpha}\left(q^{n} t\right)\right|>$ $\lambda-\varepsilon>0$. Lemma 2.1 implies that $\alpha_{i} t=k_{i} q^{-j_{i}} \pi \in Q$ with $k_{i} \neq q^{k}\left(l+\frac{1}{2}\right) \pi$ for all $k, l \in \mathbb{N}$, for $i=1, \cdots, m$. Hence $\left\{\alpha_{1}, \cdots, \alpha_{m}\right\}$ is commensurable and there is a positive integer $s$ such that

$$
\lim _{n \rightarrow \infty}\left|\phi_{\alpha}\left(q^{n} t\right)\right|=\prod_{i=1}^{m}\left|\phi\left(s n_{i} \pi\right)\right|
$$

Letting $\varepsilon \rightarrow 0$, we see that

$$
\lambda \leq \sup \left\{\prod_{i=1}^{m}\left|\phi\left(s n_{i} \pi\right)\right|: s=1,2, \cdots\right\} .
$$

On the other hand, by (1.1) $\alpha_{i}=a N_{i}$ for all $i$, and hence

$$
\alpha_{1}^{-1} N_{1}=\alpha_{2}^{-1} N_{2}=\cdots=\alpha_{m}^{-1} N_{m}
$$

Given any positive integer $s$, let $t=\alpha_{i}^{-1} s N_{i} \pi$ for $i=1, \cdots, m$. Note that $N_{i}=$ $q^{d_{i}} n_{i}$ and $\left|\phi\left(q^{n} k \pi\right)\right|=|\phi(k \pi)|$ for all $n, k \geq 1$; hence for $n \in \mathbb{N}$ we have

$$
\left|\phi_{\alpha}\left(q^{n} t\right)\right|=\prod_{i=1}^{m}\left|\phi\left(q^{n} s N_{i} \pi\right)\right|=\prod_{i=1}^{m}\left|\phi\left(s n_{i} \pi\right)\right| .
$$

Since $s$ is arbitrary, this implies that

$$
\lambda \geq \sup \left\{\prod_{i=1}^{m}\left|\phi\left(s n_{i} \pi\right)\right|: \quad s=1,2, \cdots\right\} .
$$


Proof of Theorem 1.1. We first show that $\varlimsup_{t \rightarrow \infty}|\phi(t)|=\phi(\pi)$ for all $q$. Since $\left|\phi\left(q^{n} \pi\right)\right|=\phi(\pi)>0$ for all $n$, using Corollary 2.2 with $m=1$ and $\alpha_{1}=n_{1}=1$, we have $\varlimsup_{t \rightarrow \infty}|\phi(t)|=\sup \{|\phi(s \pi)|: s=1,2, \cdots\} \geq \phi(\pi)$. For the reverse inequality it suffices to show that $|\phi(k \pi)| \leq \phi(\pi)$ for any $k \geq 1$.

If $k=q^{n}$ for some $n$, then $|\phi(k \pi)|=\phi(\pi)$. If $k$ is not a power of $q$, then for $n=1,2, \ldots$ we have $k \equiv k_{n}\left(\bmod q^{n}\right)$, where $1 \leq k_{n} \leq q^{n}-1$. Hence

$$
\cos \frac{\pi}{q^{n}} \geq\left|\cos \frac{k \pi}{q^{n}}\right| \text {. }
$$

It follows that $\phi(\pi) \geq|\phi(k \pi)|$, so that $\overline{\lim }_{t \rightarrow \infty}|\phi(t)|=\phi(\pi)$ for all $q$. From (2.1) it is easy to see that if $q$ is even then $\phi\left(q^{n} \pi\right)=-\phi(\pi)$ for all $n$, and if $q$ is odd then

$$
\phi\left(q^{n} \pi\right)=\phi(\pi) \text { if } n \text { is even, }-\phi(\pi) \text { if } n \text { is odd. }
$$

Hence Theorem 1.1 follows.

Lemma 2.3. Let $q \geq 3$ and $\alpha_{i}=a q^{d_{i}} n_{i}$ be defined by (1.1)-(1.3). If there is an integer $s \in \mathbb{N}$ such that for all $i=1, \cdots, m$,

$$
s r_{i} \not \equiv q / 2(\bmod q) \quad \text { and } \quad s r_{i} \not \equiv 0(\bmod q) \text {, }
$$

then $\varlimsup_{t \rightarrow \infty}\left|\phi_{\alpha}(t)\right|>0$. Otherwise $\lim _{t \rightarrow \infty}\left|\phi_{\alpha}(t)\right|=0$.

Proof. Suppose that $(2.5)$ is true. We will show that for all $i=1, \cdots, m$,

$$
s N_{i} \neq q^{j}(l+1 / 2) \quad \text { for all } \quad j, l \in \mathbb{N} .
$$

In fact, we have

$$
s N_{i}=q^{d_{i}}\left(s q l_{i}+s r_{i}\right)
$$

Since $s r_{i} \not \equiv 0(\bmod q)$, so $q \nmid\left(s q l_{i}+s r_{i}\right)$. If $s N_{i}=q^{j}(l+1 / 2)$ for some $j, l \in \mathbb{N}$, then by the uniqueness of the prime factorization we have $s q l_{i}+s r_{i}=q l+q / 2$. This implies that $s r_{i} \equiv q / 2(\bmod q)$, contradicting $(2.5)$. Therefore $(2.6)$ is true for all $i=1, \cdots, m$. By (2.4) we can let $t=s \alpha_{i}^{-1} N_{i} \pi, i=1, \cdots, m$; then, by (2.3) and (2.6) for all $n$

$$
\left|\phi_{\alpha}\left(q^{n} t\right)\right|=\prod_{j=1}^{m}\left|\phi\left(q^{n} s N_{j} \pi\right)\right|=\prod_{j=1}^{m}\left|\phi\left(s N_{j} \pi\right)\right|>0 .
$$

Conversely, assume that for each integer $s$, there is an $i, 1 \leq i \leq m$, such that

$$
\text { either } s r_{i} \equiv q / 2(\bmod q) \quad \text { or } \quad s r_{i} \equiv 0(\bmod q) \text {. }
$$

We will show that $\lim _{t \rightarrow \infty}\left|\phi_{\alpha}(t)\right|=0$. Suppose otherwise, by Corollary 2.2, then $\left\{\alpha_{1}, \cdots, \alpha_{m}\right\}$ is commensurable and

$$
\varlimsup_{t \rightarrow \infty}\left|\phi_{\alpha}(t)\right|=\sup \left\{\prod_{i=1}^{m}\left|\phi\left(s n_{i} \pi\right)\right|: s=1,2, \cdots\right\}>0 .
$$

Hence there exists an integer $s$ such that

$$
\prod_{i=1}^{m}\left|\phi\left(s n_{i} \pi\right)\right|>0 \text {. }
$$

Since $\left|\phi\left(q^{n} k \pi\right)\right|=|\phi(k \pi)|$, we can therefore assume that $q \nmid s$. This implies that $s r_{i} \not \equiv 0(\bmod q)$. On the other hand, by $(2.3)$, for $i=1, \cdots, m$, we have $s n_{i}=$ 
$s q l_{i}+s r_{i} \neq q^{j}(l+1 / 2)$ for any $j, l \in \mathbb{N}$. It follows that $s r_{i} \not \equiv q / 2(\bmod q)$. This contradicts $(2.7)$.

Proof of Theorem 1.2. (i) If $\varlimsup_{t \rightarrow \infty}\left|\phi_{\alpha}(t)\right|>0$, then $\left\{\alpha_{1}, \cdots, \alpha_{m}\right\}$ is commensurable by Corollary 2.2.

(ii) Assume that $\left\{\alpha_{1}, \cdots, \alpha_{m}\right\}$ is commensurable. If $q$ is odd, then (2.5) is satisfied with $s=1$. Suppose that $q$ is even. If $r_{j} \neq q / 2$ for all $j$, then (2.5) is satisfied with $s=1$. If $r_{j}=q / 2$ for some $j$, then $s r_{j}=s q / 2 \equiv 0(\bmod q)$ if $s$ is even and $s r_{j} \equiv q / 2(\bmod q)$ if $s$ is odd. By Lemma 2.3, hence $\lim _{t \rightarrow \infty}\left|\phi_{\alpha}(t)\right|=0$.

Corollary 2.3. If $q \geq 3$ and $\phi(t)=\prod_{n=1}^{\infty} \cos \left((\sqrt{q})^{n} t\right)$, then $\lim _{t \rightarrow \infty} \phi(t)=0$.

The corollary follows directly from Theorem 1.2 by writing

$$
\phi(t)=\prod_{n=1}^{\infty} \cos \left(q^{n} t\right) \cdot \prod_{n=1}^{\infty} \cos \left(\sqrt{q} \cdot q^{n} t\right)
$$

and taking $\left\{\alpha_{1}, \alpha_{2}\right\}=\{1, \sqrt{q}\}$. It is a special case of an elegant but more involved result due to Erdös and Salem [S] that for $\phi(t)=\prod_{n=1}^{\infty} \cos \left(\rho^{n} t\right), 0<\rho<1$, $\lim _{t \rightarrow \infty} \phi(t)=0$ if and only if $\rho^{-1} \neq 2$ is not a P.V. number.

To illustrate Theorem 1.1, we display the graphs of $\phi(t)$ for $q=3$ and 4 . For $q=3$, we have

$$
\varlimsup_{t \rightarrow \infty} \phi(t)=\left|\varliminf_{t \rightarrow \infty} \phi(t)\right|=\phi(\pi) \approx 0.4663
$$

For $q=4$ we have

$$
\varliminf_{t \rightarrow \infty} \phi(t)=-\phi(\pi) \approx 0.6926
$$

However, we do not have an explicit expression for $\varlimsup_{t \rightarrow \infty} \phi(t)$. It appears that $\varlimsup_{t \rightarrow \infty} \phi(t)<\phi(\pi)$.

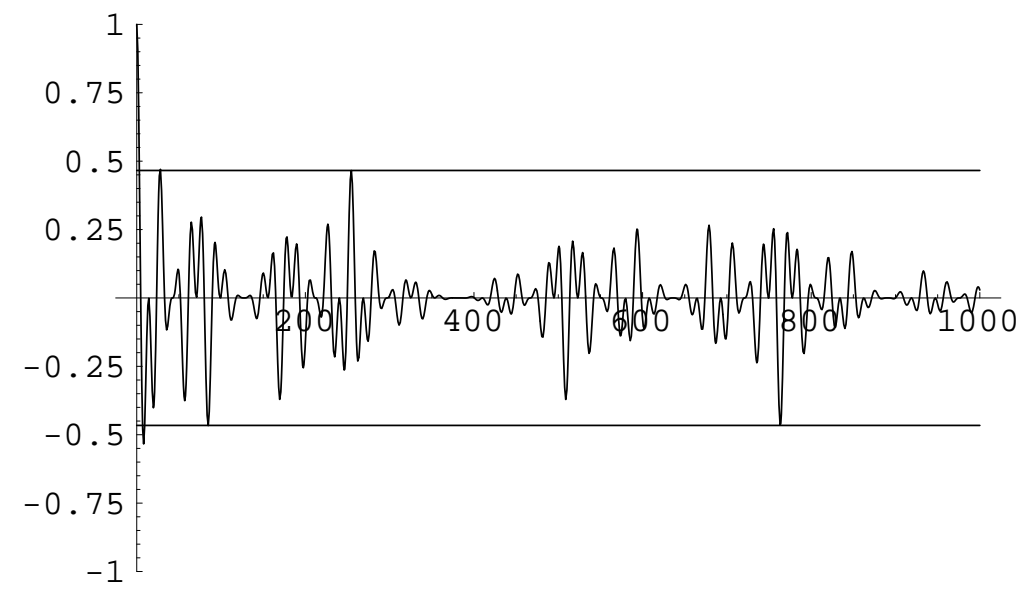

Figure 1. The graph of $\phi(t)$ for $q=3$ 




Figure 2. The graph of $\phi(t)$ for $q=4$

\section{REFERENCES}

[FL] A. H. Fan and K. S. Lau Asymptotic behavior of multiperiodic functions $G(x)=$ $\prod_{n=1}^{\infty} g\left(x / 2^{n}\right)$, J. Four. Anal. and Appl., 4 (1998), 130-150. MR 99j:41054

[LW] K. S. Lau and J. Wang, Mean quadratic variations and Fourier Asymptotics of self-similar measures, Monat. Math., 115 (1993), 99-132. MR 94g:42018; Corrigendum MR 96c:42027

[L] Q. Liu, An extension of a functional equation of Mandelbrot and Poincare, preprint.

[S] R. Salem, Algebraic numbers and Fourier transformations, Heath Math. Monographs, Boston. 1962. MR 28:1169

[Str1] R. Strichartz, Fourier asymptotics of fractal measures, J. Functional Anal., 89 (1990), 154-181. MR 91m:42015

[Str2] R. Strichartz, Self-similar measure and their Fourier transform I., Indiana University Math. J., 39 (1990), 797-817. MR 92k:42015

[WW] N. Wiener and A. Wintner, On singular distributions, J. Math. Phy., 17 (1939), 233-346.

Department of Mathematics, University of Wisconsin-Green Bay, Green Bay, WisCONSIN 54311

E-mail address: HUT@uwgb.edu

Department of Mathematics, The Chinese University of Hong Kong, Shatin, Hong KONG

E-mail address: kslau@math.cuhk.edu.hk 